\title{
Ensilado de residuos de trucha en la alimentación de ovinos de engorde
}

\author{
TROUT RESIDUES SILAGE IN THE FEEDING OF FATTENING SHEEP
}

Juan Parisuaña-Callata ${ }^{1}$, Marisol Churacutipa-Mamani ${ }^{2}$, Alberto Salas ${ }^{3}$, Maritza Barriga-Sánchez ${ }^{3}$, Marcelino J. Araníbar ${ }^{1,4}$

\section{Resumen}

Se determinó el efecto del nivel de inclusión de ensilado de residuos de trucha (ERT) en la ración de ovinos sobre el consumo medio diario (CMD), la ganancia media diaria (GMD), el índice de conversión alimenticia (ICA) y el sabor de la carne de ovinos. Se distribuyeron 30 ovinos en tres tratamientos ( 0,4 y $8 \%$ de ERT), considerando 5 réplicas por tratamiento. Al final del experimento ( 84 días) los ovinos fueron beneficiados y se realizó la prueba de degustación de la carne. Los resultados indicaron que el nivel más alto de ERT en la ración redujo el CMD $(2.08,2.01 \mathrm{vs.} 1.65 \mathrm{~kg} / \mathrm{día}$ para 0,4 y $8 \%$ de ERT, respectivamente, $\mathrm{p}<0.001)$ y la $\operatorname{GMD}(0.25,0.28$ vs $0.23 \mathrm{~kg} /$ día, $\mathrm{p}<0.03$ ), mientras que el ICA mejoró ( 8.49 vs $7.28,7.07 \mathrm{~kg}: \mathrm{kg}, \mathrm{p}<0.001)$. Además, la inclusión de ERT no afectó el sabor de la carne de ovino. Se concluye que el ERT en las raciones de engorde de ovinos mejora el rendimiento productivo sin afectar el sabor de la carne.

Palabras clave: ovinos; ensilado de trucha; rendimiento productivo; alimentación

\section{Abstract}

The effect of the level of inclusion of trout residues silage (ERT) on lamb ration on daily feed intake (CMD), daily gain (GMD), feed efficiency (ICA) and the taste of lamb meat was evaluated. Thirty lambs were distributed in three treatments $(0,4$ and $8 \%$ ERT) considering 5 replicates per treatment. At the end of the experiment ( 84 days) the lambs were slaughtered, and a taste panel evaluation was performed. The results

\footnotetext{
${ }^{1}$ Facultad de Medicina Veterinaria y Zootecnia, Universidad Nacional del Altiplano, Puno, Perú

${ }^{2}$ Arapa San Pedro San Pablo SAC, Puno, Perú

${ }^{3}$ Instituto Tecnológico de la Producción, Lima, Perú

${ }^{4}$ E-mail: maranibar@unap.edu.pe
}

Recibido: 15 de junio de 2017

Aceptado para publicación: 15 de noviembre de 2017 
showed that the highest level of ERT in the ration reduced CMD $(2.08,2.01 \mathrm{vs} .1 .65 \mathrm{~kg} / \mathrm{day}$ for 0,4 and $8 \%$ for ERT, respectively, $\mathrm{p}<0.001)$ and $\operatorname{GMD}(0.25,0.28 \mathrm{vs} 0.23 \mathrm{~kg} /$ day, $\mathrm{p}<0.03$ ), while the ICA improved (8.49 vs. $7.28,7.07 \mathrm{~kg}: \mathrm{kg}, \mathrm{p}<0.001)$. In addition, the inclusion of ERT in the ration did not affect the taste of lamb meat. It is concluded that the ERT in the fattening rations of sheep improves the productive yield without affecting the taste of the meat.

Key words: lambs; trout silage; productive performance; feeding

\section{INTRODUCCIÓN}

La región de Puno fue líder en el Perú en la producción de ovinos $\left(2088.3 \times 10^{3}\right.$ cabezas) en 2012 (INEI, 2013) y en la extracción de trucha fresca $\left(34.2 \times 10^{3} \mathrm{t}\right)$ en 2015 (PRODUCE, 2016). Se estima que la comercialización de trucha eviscerada genera alrededor de 55\% de residuos en Puno, produciéndose alrededor de 1567.8 t mensuales de residuos altamente perecibles.

Una alternativa de manejo de los residuos de trucha es su tratamiento y conservación mediante fermentación anaerobia bacteriana (denominado ensilado), convirtiéndolos en un producto nutritivo y bastante estable (Fagbenro y Jauncey, 1993; Feltes et al., 2010). En varios países se utiliza los ensilados de pescado como materia prima en la formulación de alimentos, fundamentalmente en aves (Al-Marzooqi et al., 2010), cerdos (Calderón et al., 2017) y rumiantes (Yamamoto et al., 2007; Rahmi et al., 2008) y en la mayoría de los casos con resultados satisfactorios.

Por otro lado, los ovinos son rumiantes que tienen la capacidad de transformar alimentos bastante groseros (forrajes, pastos y subproductos agroindustriales) en productos de alto valor biológico (carne y leche). Sin embargo, cuando se realiza el engorde de estos animales, los alimentos deben tener un alto contenido de nutrientes en poco volumen y ser administrados ad libitum para que además de cubrir sus requerimientos nutricionales, permitan un mayor rendimiento productivo (NRC, 2007).
La harina de pescado es pocas veces incluida en las raciones de rumiantes debido a su alto costo. No obstante, se ha demostrado que la inclusión de ensilado de pescado en raciones fibrosas incrementa la fuente de proteína y energía en el alimento de ovinos (Díaz y Rodríguez, 2008), mejora el peso vivo y las características de la carne (Rahmi et al., 2008) y reduce la producción de metano ruminal (Barroga et al., 2001; Fievez et al., 2003).

El objetivo de la presente investigación fue determinar el efecto del nivel de inclusión de ensilado de residuos de trucha en la ración al imenticia de engorde de ovinos sobre sus parámetros productivos (ganancia diaria, consumo dealimento y conversión alimenticia) y sabor de la carne.

\section{Materiales y Métodos}

\section{Lugar e Instalaciones}

La investigación se realizó en el distrito de Arapa, departamento de Puno, Perú, a $3880 \mathrm{~m}$ de altitud. Se utilizaron 30 ovinos machos cruzados (criollo x Corriedale) de 10 meses de edad, provenientes del Centro de Investigación y Producción (CIP) Chuquibambilla, Facultad de Medicina Veterinaria y Zootecnia, Universidad Nacional del Altiplano (Puno).

Los ovinos fueron distribuidos en 15 corrales de $4 \mathrm{~m}^{2} \mathrm{c} / \mathrm{u}$. Los tratamientos incluyeron una ración control $(0 \%$ de ensilado de residuos de trucha [ERT]) y dos raciones 
experimentales ( 4 y $8 \%$ de ERT), y cada tratamiento fue replicado 5 veces (un corral con dos ovinos por réplica). Asimismo, los animales fueron desparasitados antes de iniciar el experimento con Destroyer TF ${ }^{\circledR}$ (triclabendazole $12 \mathrm{~g}$, febendazole $7.5 \mathrm{~g}$ ) por vía oral, a una dosis de $1 \mathrm{ml} / 12 \mathrm{~kg}$ de peso corporal. La utilización de los animales en experimentación contó con la aceptación del comité de ética de la institución.

Los ovinos tuvieron 30 días de acostumbramiento (periodo pre-experimental). El periodo experimental fue de 84 días y los controles de los parámetros productivos se realizaron cada 28 días.

\section{Raciones Alimenticias}

Los ingredientes utilizados en las raciones fueron adquiridos del mercado local, a excepción del ERT, que fue elaborado en las instalaciones de la Empresa Arapa SAC, Puno, Perú. La técnica de fermentación del ERT fue desarrollada por el Instituto Tecnológico de la Producción (ITP) con el uso de «koji» (Aspergillus oryzae y Saccharomyces cerevisae), arroz y melaza. El valor de $\mathrm{pH}$ del ERT fue $\leq 4.5$ y los valores de histamina fueron $<85 \mathrm{mg} / \mathrm{kg}$. El ERT contenía $61.3 \%$ humedad, $12.0 \%$ de proteína bruta, $21.5 \%$ de grasa bruta y $5.3 \%$ de cenizas (Churacutipa, 2016).

Se consideró la tabla de alimentos FEDNA (2003) y las tablas de composición de alimentos y requerimientos nutricionales de ovinos de la NRC (2007) en la formulación de las tres raciones para engorde $(0,4$ y $8 \%$ de ERT). Las raciones (Cuadro 1) fueron formuladas a mínimo costo con la ayuda del programa computacional AEZO-FD II (AEZO, 2003).

En la elaboración de las raciones, el ERT se administró haciendo la equivalencia al 90\% de materia seca (MS); es decir, 10.8\% de ERT semilíquido fue equivalente a $4 \%$ de inclusión en la ración, mientras que $20.2 \%$ de ERT semilíquido fue equivalente a $8 \%$ de la inclusión de ERT en la ración. Primero se realizó la mezcla del ERT líquido con el afrecho de trigo (Hossain y Alam, 2015) y luego fueron mezclados con las otras materias primas, con la finalidad de lograr uniformidad en la distribución de las partículas durante el mezclado de la ración.

Las raciones fueron administradas a los animales en forma diaria y ad libitum, en una forma molida grosera. Todos los animales tuvieron acceso libre al agua.

\section{Análisis Químico de los Alimentos}

Las muestras fueron analizadas por triplicado y considerando las recomendaciones de la AOAC (2011). La materia seca fue determinada por el método gravimétrico, la proteína bruta por el método Kjeldahl (contenido de $\mathrm{N} \times 6.25$ ), el contenido de grasa bruta por el método de extracción de Soxhlet y las cenizas por incineración de las muestras $\left(550{ }^{\circ} \mathrm{C}\right.$ durante 12 a $\left.20 \mathrm{~h}\right)$.

\section{Parámetros Productivos}

El control de peso vivo y el peso del alimento balanceado fue realizado cada 28 días con una balanza electrónica OHAUS ${ }^{\circledR}$ T32XW, con una capacidad de 300 y una precisión de $0.1 \mathrm{~kg}$. El peso vivo al inicio del experimento fue de $37.2 \pm 1.1,36.0 \pm 0.8 \mathrm{y}$ $36.3 \pm 0.9 \mathrm{~kg}$ para los tratamientos control, 4 y $8 \%$ de ERT, respectivamente.

La ganancia media de peso por día (GMD) se determinó considerando el peso vivo alcanzado en cada periodo entre los días experimentales ( $\mathrm{kg} / \mathrm{día})$. El consumo medio de alimento por día (CMD) se determinó considerando el consumo total realizado en cada periodo entre los días experimentales ( $\mathrm{kg} /$ día). La conversión alimenticia se determinó mediante la relación entre el consumo de alimento por día y la ganancia de peso por día (CMD / GMD) de los ovinos en cada periodo (el valor fue expresado en unidades de alimento utilizado para producir una unidad de peso vivo del animal [kg:kg]). 
Cuadro 1. Composición de las raciones experimentales empleando ensilado de residuos de trucha para ovinos en engorde

\begin{tabular}{lccc}
\hline \multirow{2}{*}{ Ingredientes } & \multicolumn{3}{c}{ Tratamientos (\% de ensilado) } \\
\cline { 2 - 4 } & 0 & 4 & 8 \\
\hline Heno de avena molida & 33.05 & 38.77 & 43.39 \\
Afrecho de trigo & 54.95 & 45.03 & 36.73 \\
Pasta de algodón & 8.35 & 8.32 & 8.31 \\
Ensilado de residuos de trucha ${ }^{1}$ & 0.00 & 4.44 & 8.31 \\
Melaza de caña & 1.70 & 1.75 & 1.66 \\
Carbonato de calcio & 1.35 & 1.09 & 1.00 \\
Sal común & 0.50 & 0.50 & 0.50 \\
Premezcla de vitaminas y minerales ${ }^{2}$ & 0.10 & 0.10 & 0.10 \\
\hline Total & 100.00 & 100.00 & 100.00 \\
\hline Nutrientes calculados & & & \\
Energía metabolizable, Mcal/kg & 2.44 & 2.44 & 2.43 \\
Proteína bruta, \% & 13.05 & 13.03 & 13.04 \\
Proteína degradable rumen, \% & 16.35 & 15.28 & 14.26 \\
Fibra detergente neutro, \% & 33.89 & 32.43 & 31.16 \\
Calcio, \% & 0.70 & 0.69 & 0.72 \\
Fósforo total, \% & 0.68 & 0.65 & 0.63 \\
\hline Análisis proximal (en $M S)^{3}$ : & & & \\
Materia seca, \% & 96.5 & 96.9 & 95.7 \\
Proteína bruta, \% & 13.7 & 12.9 & 13.2 \\
Grasa bruta, \% & 6.9 & 10.2 & 11.6 \\
Cenizas, \% & 6.9 & 6.8 & 6.7 \\
\hline
\end{tabular}

${ }^{1}$ El ERT presentó $89 \%$ de digestibilidad in vitro de la proteína

${ }^{2}$ Por cada kilogramo de alimento se adicionó 10000 UI de Vit A, 1000 UI de Vit D3, 10 UI de Vit E, 0.1 $\mathrm{mg}$ de cobalto, $10 \mathrm{mg}$ de cobre, $40 \mathrm{mg}$ de hierro, $30 \mathrm{mg}$ de manganeso, $0.1 \mathrm{mg}$ de selenio, $2 \mathrm{mg}$ de yodo, $50 \mathrm{mg}$ de zinc y $50 \mathrm{mg}$ de magnesio

${ }^{3} \mathrm{MS}=$ materia seca

\section{Beneficio y Muestras de Carne}

Dos animales por tratamiento se beneficiaron al final del experimento para determinar el efecto de la inclusión de ERT en la ración sobre el sabor de la carne. El beneficio de las ovejas se realizó mediante una incisión a nivel de la articulación atlantooccipital, para anular los mecanismos sensitivos del animal, y seguidamente se realizó el sangrado, desollado y eviscerado del animal.
Se dejó madurar la carne durante 24 horas, luego se almacenó bajo refrigeración a $5 \pm 1^{\circ} \mathrm{C}$ en la planta Arapa San Pedro y San Pablo SAC. Se separaron $4 \mathrm{~kg}$ de masa muscular (lomo y muslo) de cada animal y se realizó un pool de $8 \mathrm{~kg}$ por tratamiento, para volver a separar en dos porciones de $4 \mathrm{~kg}$ por tratamiento. Una muestra por tratamiento se quedó en Arapa y las otras fueron remitidas en forma refrigerada, por vía aérea, al laboratorio del ITP, en una caja de poliestireno expandido con hielo. 


\section{Análisis Sensorial}

Se realizaron dos evaluaciones sensoriales: una en Puno y otra en Lima, el mismo día y a la misma hora. En Arapa, Puno, participaron 18 personas no entrenadas, mientras que en el ITP en Lima se tuvo un panel de 18 evaluadores entrenados.

En la prueba sensorial realizada en Arapa participaron personas adultas, hombres y mujeres entre 20 y 65 años. En el ITP los evaluadores tenían entre 35 y 55 años. Previo a la prueba, se informó a los participantes de la escala empleada en la ficha sensorial. Asimismo, se les pidió evitar conversaciones entre ellos y evitar expresiones durante la realización de la prueba. Además, se les pidió que anotaran cualquier percepción anormal del sabor.

Las muestras fueron cortadas en trozos de $70 \mathrm{~g}$ y cocinadas en la presentación de carne sancochada. Las muestras fueron presentadas en platos con códigos para cada tratamiento. Mediante esta prueba se deseaba establecer posibles diferencias en el agrado entre las muestras, así como analizar la magnitud de las diferencias aplicando una modificación a la prueba de comparaciones apareadas de Scheffé (Anzaldúa-Morales, 1994).

Las muestras del grupo control fueron comparadas con las muestras provenientes de los tratamientos con 4 y $8 \%$ de ERT en una escala de 1 a 7 puntos: 7: sabor mucho mejor que la muestra control; 6 : sabor moderadamente mejor que la muestra control; 5: sabor levemente mejor que la muestra control; 4: sabor igual que la muestra control; 3 : sabor levemente menor que la muestra control; 2: sabor moderadamente menor que la muestra control; 1: sabor bastante menor que la muestra control.

\section{Análisis Estadístico}

El análisis estadístico fue realizado usando el procedimiento de modelo lineal general (GLM) del programa estadístico SAS (2004).
Los datos de peso vivo, ganancia media diaria, consumo de alimento y conversión alimenticia fueron sometidos al análisis de medias por mínimos cuadrados (LS Means) en un diseño completamente al azar con tres tratamientos y cinco repeticiones por tratamiento (Littell et al., 2002). Asimismo, para el análisis de sabor de la carne se usó el programa Minitab $^{\circledR}$. Las diferencias fueron consideradas significativas si $\mathrm{p}<0.05$.

\section{Resultados y Discusión}

\section{Parámetros Productivos}

Los resultados de peso vivo y ganancia media diaria de peso se presentan en los cuadros 2 y 3 . A los 28,56 y 84 días del experimento se observó un efecto benéfico de los tratamientos sobre el comportamiento productivo $(\mathrm{p}<0.05)$. En general, los animales a los 84 días de recibir el ERT al 4\% en la ración obtuvieron mejores resultados que aquellos que recibieron $8 \%$ de ERT $(\mathrm{p}<0.05)$, aunque sin diferencia significativa con el grupo control.

En los dos primeros periodos (0-28 y 2956 días) hubo diferencias entre tratamientos con respecto a la ganancia media diaria $(\mathrm{p}<0.05)$, no así en el último periodo (57-84 días). No obstante, considerando el total de la fase experimental ( 0 a 84 días) se observa que los animales del tratamiento que recibieron $4 \%$ de ERT en la ración tuvieron una mayor ganancia de peso que el grupo que recibió $8 \%$ de ERT (Cuadro 2). Por otro lado, el consumo de alimento varió significativamente entre grupos según el periodo experimental, pero considerando la fase de engorde completa de 0-84 días se tuvo que el consumo de alimento disminuyó con el nivel de $8 \%$ ERT en la ración $(\mathrm{p}<0.001)$, pero fue similar entre el grupo control y el grupo con $4 \%$ de ERT.

La conversión alimenticia fue mejor en el periodo de 0-28 días en el tratamiento de $4 \%$ de ERT $(\mathrm{p}<0.02)$, mientras que en el pe- 
Cuadro 2. Efecto de la inclusión de ensilado de residuos de trucha (ERT) sobre el peso vivo medio $(\mathrm{kg})$ de los ovinos a los 28,56 y 84 días experimentales

\begin{tabular}{lccc}
\hline Tratamientos & 28 días & 56 días & 84 días \\
\hline Control & $43.3^{\mathrm{b}} \pm 1.36$ & $50.8^{\mathrm{a}} \pm 1.57$ & $57.9^{\mathrm{ab}} \pm 1.65$ \\
ERT, $4 \%$ & $45.1^{\mathrm{a}} \pm 0.89$ & $51.4^{\mathrm{a}} \pm 1.84$ & $59.3^{\mathrm{a}} \pm 1.54$ \\
ERT, $8 \%$ & $42.1^{\mathrm{b}} \pm 0.76$ & $47.8^{\mathrm{b}} \pm 1.06$ & $56.0^{\mathrm{b}} \pm 1.99$ \\
\hline Probabilidad & 0.006 & 0.006 & 0.035 \\
\hline
\end{tabular}

a,b Superíndices diferentes dentro de columnas difieren estadísticamente $(p<0.05)$

Cuadro 3. Efecto de la inclusión de ensilado de residuos de trucha (ERT) sobre la ganancia de peso ( $\mathrm{kg} /$ día), consumo de alimento ( $\mathrm{kg} /$ día) y conversión alimenticia de los ovinos durante 84 días experimentales

\begin{tabular}{|c|c|c|c|c|c|}
\hline $\begin{array}{l}\text { Parámetro } \\
\text { productivo }\end{array}$ & Tratamiento & $0-28$ & $29-56$ & $57-84$ & $0-84$ \\
\hline \multirow{3}{*}{$\begin{array}{l}\text { Ganancia } \\
\text { media diaria, } \\
\mathrm{kg} / \text { día }\end{array}$} & Control & $0.21^{b} \pm 0.04$ & $0.27^{a} \pm 0.04$ & $0.26 \pm 0.03$ & $0.25^{\mathrm{ab}} \pm 0.02$ \\
\hline & ERT, $4 \%$ & $0.32^{\mathrm{a}} \pm 0.05$ & $0.23^{\mathrm{ab}} \pm 0.04$ & $0.28 \pm 0.03$ & $0.28^{\mathrm{a}} \pm 0.02$ \\
\hline & ERT, $8 \%$ & $0.22^{\mathrm{b}} \pm 0.05$ & $0.19^{\mathrm{b}} \pm 0.05$ & $0.29 \pm 0.04$ & $0.23^{b} \pm 0.03$ \\
\hline \multirow{3}{*}{$\begin{array}{l}\text { Consumo } \\
\text { medio diario, } \\
\text { kg/día }\end{array}$} & Control & $1.68^{\mathrm{b}} \pm 0.15$ & $2.13^{a} \pm 0.12$ & $2.44^{\mathrm{a}} \pm 0.14$ & $2.08^{\mathrm{a}} \pm 0.07$ \\
\hline & ERT, 4\% & $1.91^{\mathrm{a}} \pm 0.04$ & $1.76^{\mathrm{b}} \pm 0.21$ & $2.35^{\mathrm{a}} \pm 0.10$ & $2.01^{\mathrm{a}} \pm 0.10$ \\
\hline & ERT, 8\% & $1.52^{\mathrm{b}} \pm 0.07$ & $1.56^{\mathrm{b}} \pm 0.10$ & $1.86^{\mathrm{b}} \pm 0.16$ & $1.65^{b} \pm 0.09$ \\
\hline \multirow{3}{*}{$\begin{array}{l}\text { Índice de } \\
\text { conversión } \\
\text { alimenticia, } \\
\text { kg:kg }\end{array}$} & Control & $8.01^{\mathrm{a}} \pm 1.25$ & $7.97 \pm 1.19$ & $9.49^{\mathrm{a}} \pm 0.73$ & $8.49^{\mathrm{a}} \pm 0.37$ \\
\hline & ERT, $4 \%$ & $6.01^{b} \pm 0.77$ & $7.75 \pm 0.88$ & $8.42^{\mathrm{a}} \pm 1.12$ & $7.28^{b} \pm 0.37$ \\
\hline & ERT, $8 \%$ & $6.95^{\mathrm{ab}} \pm 0.75$ & $8.33 \pm 1.57$ & $6.41^{b} \pm 0.57$ & $7.07^{b} \pm 0.47$ \\
\hline
\end{tabular}

a,b Superíndices diferentes dentro de columnas y por parámetro productivo difieren estadísticamente $(p<0.05)$

riodo de 57-84 días fue mejor en el tratamiento de $8 \%(\mathrm{p}<0.001)$ (Cuadro 3). Sin embargo, al considerar el periodo completo de engorde (0-84 días), la conversión fue similar con la inclusión de 4 y $8 \%$ de ERT en la ración, pero mejor que en el grupo control $(p<0.001)$, pese al menor consumo de alimento que tuvieron los animales con el mayor nivel de ERT. Los resultados confirman que aun cuando los animales consumieron menos alimento, mejoraron la conversión alimenticia con la inclusión del ERT, lo que podría deberse a la alta digestibilidad mostrada in vitro de la proteína (89\%) en el ERT (según el análisis realizado) y también a la presencia de los ácidos grasos poliinsaturados en el ERT. De hecho, se ha reportado que la inclusión de aceite de pescado en el alimento reduce la formación de metano y aumenta la producción de ácido propiónico en el rumen (Fievez et al., 2003), siendo este último un importante metabolito para el proceso de gluconeogénesis en ru- 
Cuadro 4. Estadísticos descriptivos de las calificaciones del sabor de la carne de ovino (18 observaciones por $\operatorname{lugar}^{1} \mathrm{y}$ por tratamiento)

\begin{tabular}{cccc}
\hline $\begin{array}{c}\text { Lugar, } \\
\text { \% ERT }\end{array}$ & Mínimo & Máximo & Media \\
\hline Arapa, 4 & 2 & 7 & $5.1 \pm 1.5$ \\
Arapa, 8 & 3 & 7 & $4.3 \pm 1.4$ \\
ITP, 4 & 2 & 7 & $4.0 \pm 1.4$ \\
ITP, 8 & 2 & 7 & $4.6 \pm 1.4$ \\
\hline
\end{tabular}

${ }^{1}$ Arapa (Arapa San Pedro San Pablo SAC, Puno); ITP (Instituto Tecnológico de la Producción, Lima)

miantes. Esta afirmación ha sido relacionada con cambios en las proporciones de los microorganismos ruminales (Vargas et al., 2017).

La inclusión de 4\% de ERT en la ración favoreció el incremento de peso y el consumo de alimento con relación a la inclusión de $8 \%$ de ERT. Estos resultados son consistentes con Rahmi et al. (2008), quienes demostraron que ovejas alimentadas con ensilado de pescado mostraron mayor peso vivo, pero cuando el ensilado fue adicionado a un nivel del $50 \%$ de la ración se disminuyó considerablemente el consumo de alimento. Asimismo, Díaz y Rodríguez (2008) demostraron que la suplementación con ensilado de pescado a carneros alimentados con henos tropicales aumenta el consumo voluntario de la materia seca y proteína, pero reduce el consumo de fibra detergente neutro y aumenta la digestibilidad de la materia seca y proteína. También ha sido indicado que la inclusión de ensilado proveniente de peces de agua dulce produce una mejor conversión alimenticia en ovinos que aquel proveniente de peces de agua salada (Yamamoto et al., 2007). Por otro lado, Barroga et al. (2001) demostraron que la inclusión de ensilado de residuos de
Seriola quinqueradiata en la ración mejora el aprovechamiento de la proteína y energía, acompañado de una aparente reducción en la producción de metano, lo que proveería energía para sostener el crecimiento de los ovinos.

La retención de nitrógeno por los ovinos está relacionada con su nivel de ingestión y el nivel de energía en la ración; es decir, contenidos altos de nitrógeno en la ración tienen menor retención corporal cuando la ración contiene niveles bajos de energía. Sin embargo, niveles acordes a los requerimientos producen una retención positiva de nitrógeno en el animal (NRC, 2007). De hecho, Shqueir et al. (1984) encontraron un incremento en la retención de nitrógeno de $0.5,0.5,0.6 \mathrm{y}$ $0.7 \mathrm{~g} / \mathrm{kg}$ PV0.75 cuando incrementaron la cantidad de ensilado de pescado $(0,5,10 \mathrm{y}$ $15 \%$, respectivamente) en la ración de ovinos. Consistentemente, Samuels et al. (1991) encontraron, también, mayor retención de nitrógeno en los ovinos cuando el ensilado de residuos de pescado fue adicionado a alimentos con alto contenido en fibra cruda. Últimamente ha sido reportado que las raciones concentradas en energía reducen la producción de metano y mejoran la eficiencia productiva de los ovinos (Paganoni et al., 2017).

El ensilado de pescado también ha sido utilizado como fuente de proteína en la alimentación de terneros recién destetados, pues por su edad aprovechan la disponibilidad de proteína y aminoácidos del ensilado (Winter y Javed, 1980). La inclusión de ensilado a los alimentos fibrosos proporciona mayores nutrientes y aumenta su disponibilidad (Samuels et al., 1991); sin embargo, debido al contenido de agua y elevado costo de transporte del ensilado, la tendencia actual es la preparación de alimentos fibrosos enriquecidos secos (Hossain y Alam, 2015) para rumiantes adultos. Por otro lado, una disminución en la degradación de la proteína de los ensilados en el rumen puede lograrse con el tratamiento con formalina, obteniéndose mayor proteína a nivel intestinal y, por lo tanto, mayor disponibilidad de aminoácidos para el 
crecimiento del animal (Husain y Offer, 1987).

\section{Análisis Sensorial de la Carne}

Los resultados del análisis sensorial de la carne se muestran en el Cuadro 4. En Arapa, el tratamiento con $8 \%$ de ERT fue calificado como similar al grupo testigo (calificación 4), obteniendo el tratamiento con $4 \%$ de ERT la mejor calificación, aunque sin diferencias significativas; mientras que en el ITP los tres tratamientos fueron considerados como similares.

La inclusión de 8\% de ERT en la ración no afectó el sabor de la carne de los ovinos. En este mismo sentido, Shqueir et al. (1984) reportaron que la inclusión de ensilado de pescado (merluza del Pacífico entera más 10\% de vísceras de atún) en la ración no tuvo efecto perjudicial sobre el sabor de la carne de ovinos en comparación al tratamiento control. También Rahmi et al. (2008) encontraron que la carne de ovinos alimentados con ensilado de pescado mejoraba la calidad y la forma de la canal. En un estudio reciente, Calderón et al. (2017) indican que la inclusión de hasta 10\% de ERT en la ración no afecta el sabor de la carne de cerdos; no obstante, Al-Marzooqi et al. (2010) señalan que niveles de 30\% de inclusión en la ración provocaron sabor a pescado en la carne de pollos.

\section{Conclusiones}

- La inclusión de 4 y $8 \%$ de ensilado de residuos de trucha en la ración alimenticia de engorde de ovinos mejora la ganancia de peso y la conversión alimenticia.

- La inclusión de 4 y $8 \%$ de ensilado de residuos de trucha en la ración alimenticia de engorde de ovinos no cambia el sabor de la carne de ovinos.

- Los resultados sugieren que el ensilado de residuos de trucha se presenta como una alternativa viable y potencial en la alimentación y engorde de ovinos.

\section{Agradecimiento}

Los autores agradecen a INNOVATEPERÚ por el financiamiento del Proyecto PIPEI-6-P-024-005-13, así como a la empresa ARAPA San Pedro y San Pablo SAC y al Instituto Tecnológico de la Producción por las facilidades prestadas para el desarrollo del estudio.

\section{Literatura Citada}

1. [AEZO] Asociación de Estudiantes de Zootecnia. 2003. Programa computacional de balanceo de raciones para animales FD-II. Pontificia Universidad Católica de Chile. Santiago de Chile.

2. Al-Marzooqi W, Al-Farsi MA, Kadim IT, Mahgoub O, Goddard JS. 2010. The effect of feeding different levels of sardine fish silage on broiler performance, meat quality and sensory characteristics under closed and open-sided housing systems. Asian Australas J Anim Sci 23: 1614-1625. doi: 10.5713/ ajas.2010.10119

3. Anzaldúa-Morales A. 1994. La evaluación sensorial de los alimentos en la teoría y la práctica. Zaragoza, España: Ed Acribia. 198 p.

4. [AOAC] Association of Official Analytical Chemists International. 2011. Official methods of analysis of AOAC International. $18^{\text {th }}$ ed. $4^{\text {th }}$ Rev. Gaitheresburg, SA: AOAC International. 1965 p.

5. Barroga JA, Pradhan R, Tobioka $H$. 2001. Evaluation of fish silage-sweet potato mixed diet with Italian ryegrass silage as basal ratio non-nitrogen utilization and energy balance in growing lambs. Anim Sci J 72: 189-197.

6. Calderón-Quispe V, ChuracutipaMamani M, Salas A, Barriga-Sánchez M, Araníbar MJ. 2017. Inclusión de ensilado de residuos de trucha en el alimento de cerdos y su efecto en el rendimiento productivo y sabor de la carne. 
Rev Inv Vet Perú 28: 265-274. doi: 10.15381/rivep.v28i2.13055

7. Churacutipa-Mamani M. 2016. Obtención de un ensilado biológico a partir de residuos de trucha (Oncorhynchus mykiss). Tesis de Ingeniero Agroindustrial. Puno, Perú: Univ. Nacional del Altiplano. $103 \mathrm{p}$.

8. Díaz-Ríos HL, Rodríguez-Carias AA. 2008. Consumo voluntario y digestibilidad de nutrientes de heno de gramíneas y leguminosas tropicales suplementadas con ensilaje de residuos de pescadería. J Agric Univ PR 92(1-2): 27-38.

9. Fagbenro O, Jauncey K. 1993. Chemical and nutritional quality of stored fermented fish (tilapia) silage. Bioresource Technol 46: 207-211. doi: 10.1016/0960-8524(93)90121-Q

10. [FEDNA] Fundación Española para el Desarrollo de la Nutrición Animal. 2003. Normas FEDNA para la formulación de piensos compuestos. $2^{\mathrm{a}}$ ed. De Blas C, Mateos GG, Rebollar PG (eds). Madrid, España: FEDNA. 423 p.

11. Feltes CMM, Correia GFJ, Beirao HL, Block MJ, Ninow LJ, Spiller RV. 2010. Alternativas para a agregação de valor aos residuos da industrialização de peixe. Rev Bras Eng Agríc Ambient 14: 669-677. doi: 10.1590/S1415-43662010000600014

12. Fievez, V, Dohme F, Danneels M, Raes K, Demeyer D. 2003. Fish oils as potent rumen methane inhibitors and associated effects on rumen fermentation in vitro and in vivo. Anim Feed Sci Technol 104: 41-58. doi: 10.1016/S0377-8401(02)00330-9

13. Hossain U, Alam NMKA. 2015. Production of powder fish silage from fish market wastes. SAARC J Agric 13(2): 13-25.

14. Husain RAK, Offer NW. 1987. Effect of formaldehyde treatment on the degradation of acid preserved fish silage protein in vitro. Anim Feed Sci Technol 16: 297-304. doi: 10.1016/03778401(87)90018-6
15. [INEI] Instituto Nacional de Estadística e Informática. 2013. Resultados definitivos. IV Censo Nacional Agropecuario 2012. [Internet]. Disponible en: http://proyectos.inei.gob.pe/web/ DocumentosPublicos/ResultadosFinalesIVCENAGRO.pdf

16. Littell RC, Stroup WW, Freund RJ. 2002. SAS for Linear Models. $4^{\text {th }}$ ed. Cary, NC, USA: SAS Inst. 329 p.

17. [NRC] National Research Council, 2007. Nutrient requirements of small ruminants: sheep, goats, cervids, and new world camelids. Washington DC, USA: NRC. 384 p.

18. Paganoni B, Rose G, Macleay C, Jones C, Brown DJ, Kearney G, Ferguson M, Thompson AN. 2017. More feed efficient sheep produce less methane and carbon dioxide when eating high-quality pellets. J Anim Sci 95: 38393850. doi: $10.2527 /$ jas2017.1499

19. [PRODUCE] Ministerio de la Producción. Perú. 2016. Anuario Estadístico Pesquero y Acuícola. [Internet]. Disponible en: http://www.produce.gob.pe/documentos/estadisticas/anuarios/anuarioestadistico-pesca-2015.pdf

20. Rahmi M, Faid M, ElYachioui M, Berny EH, Fakir M, Ouhssine M. 2008. Protein rich ingredients from fish waste for sheep feeding. Afr J Microbiol Res 2: 73-77.

21. Samuels WA, Fontenot JP, Allen VG, Abazinge MDA. 1991. Seafood processing wastes ensiled with straw: utilization and intake by sheep. J Anim Sci 69: 4983-4992.

22. SAS Institute Inc. 2004. SAS/STAT ${ }^{\circledR}$ 9.1 User's Guide. Cary, NC, USA: SAS Institute. $5121 \mathrm{p}$.

23. Shqueir AA, Church DC, Kellems RO. 1984. Evaluation of liquefied fish in digestibility and feedlot performance studies with sheep. Can J Anim Sci 64: 889-898.

24. Vargas JE, Andrés S, Snelling TJ, LópezFerreras L, Yáñez-Ruíz DR, GarcíaEstrada C, López S. 2017. Effect of 
sunflower and marine oils on ruminal microbiota, in vitro fermentation and digesta fatty acid profile. Front Microbiol 8: 1124. doi: 10.3389/fmicb.2017.01124

25. Winter KA, Javed AH. 1980. Fish silage as a protein source for early weaned calves. Can J Anim Sci 60: 787-789.
26. Yamamoto SM, García da Silva A, Vidotti RM, Homem AC, BonilhaPinheiro RS, Buzzulini C. 2007. Desempenho e digestibilidade dos nutrientes em cordeiros alimentados com dietas contendo silagem de residuos de peixe. R Bras Zootec 36: 1131-1139. doi: 10.1590/S1516-35982007000500021 